\title{
COGNITIVE AND PSYCHOMOTOR CORRELATES OF HAZARD PERCEPTION ABILITY AND RISKY DRIVING
}

\author{
Nebi Sümer \\ Middle East Technical University \\ Ankara, Turkey \\ Email: nsumer@metu.edu.tr
}

\begin{abstract}
Summary: Deficits in specific cognitive and psychomotor capacities, such as attention, reaction time, memory, and hand-eye coordination ability are associated with increased crash risk, especially among older drivers (e.g., Anstey et al., 2005). Higher-order cognitive processes are also closely linked with hazard perception skills (e.g., Groeger, 2000). Employing a large professional driver sample, this study examines cognitive and psychomotor correlates of driver hazard perception detection accuracy (HPDA) and risky driving. Professional drivers ( $\mathrm{N}=2541)$ who applied to psycho-technical driver assessment centers were administered a number of computer-based measures of hazard perception, monotonous and selective attention, reasoning, visual pursuit, visual perception, hand-eye coordination, and reaction time. They also completed a self-reported measure of aberrant driver behaviors and reported the accidents involved and violation tickets taken in last three years. Results showed that attention capacity and psychomotor abilities were consistently associated with HPDA. Regression analyses revealed that selective attention, reasoning ability, and visual pursuit were moderately strong predictors of HPDA. These variables, however, weakly but significantly predicted the indicators of risky driving including aberrant driving behaviors, road traffic accidents, and violation tickets. These results suggest that hazard perception skills mediate the link between specific cognitive/perceptual skills and risky driving. The findings underscore the role of higher-order perceptual and cognitive processes, especially selective attention and reasoning, underlying hazard perception ability and have implications for driver assessment, training, and cognitive demands for driving.
\end{abstract}

\section{INTRODUCTION}

Driving task can best be modeled as an interactive function of cognitive, perceptual, and motor processes. Past studies have consistently shown that deficits in specific cognitive, perceptual, and psychomotor capacities, such as selective attention, reaction time, memory, visual pursuit, and hand-eye coordination ability are associated with increased crash risk, especially among older drivers (e.g., Anstey et al., 2005). Recent studies have also shown that higher-order cognitive processes are critical for the development of hazard perception skills (e.g., Groeger, 2000). Hazard perception is defined as the ability to anticipate dangerous and risky situations in the driving environment (see Deery, 1999). Accurately detecting a looming hazard while driving is a critical stage in hazard perception process, even if hazard perception involves more than merely perceiving a hazard. According to Groeger (2000), four processes play a role in hazard perception; namely, accurately detecting the hazard, appraising the magnitude of the threat, selecting appropriate actions to avert the hazard, and finally implementation of actions chosen. 
Past research has consistently demonstrated that insufficient skills in detecting hazards play an important role in the occurrence of crashes and as compared to experienced drivers, novice drivers fail in each stage of the hazard perception process (e.g., Horswill \& McKenna, 2004). Drivers develop an effective and predictive mental model of the driving environment via driving experience and practice, and thus, develop the ability for hazard perception (McKenna \& Horswill, 1999). Furthermore, those who have better cognitive and psychomotor capacity, such as young drivers, are expected to form a more effective mental model of potential hazards and can cope better with hazardous situations than those with limited and/or deteriorating psychomotor capacity, such as elderly drivers (e.g., Horswill et al., 2009).

Recently, it has been suggested that hazard perception can be seen equivalent to situational awareness in driving. Both concepts basically describe how drivers are aware of elements in the driving environment and how they use this awareness to predict hazardous situations (Helman, 2008). Situational awareness is a critical element of detecting a developing hazard accurately and both situational awareness and hazard perception are accepted to directly link with higher order cognitive abilities (Banbury \& Tremblay, 2004; Horswill \& McKenna, 2004). Therefore, there is a need to assess specific cognitive as well as psychomotor correlates of hazard perception detection accuracy, which have been understudied in previous research with few exceptions (e.g., Horswill et al., 2008). Hence, this study aims to examine the power of cognitive and psychomotor skills in predicting HPDA and risky driving among a large professional driver sample.

HPDA demands higher order-cognitive abilities, especially the ability to orient attentional control (Fitzgerald \& Harrison, 1999), which is responsible for regulating critical processes in preventing potential crashes on the road. Furthermore, the fundamental processes underlying HPDA are closely linked with driver's ability in visual monitoring, perception, and cognitive detection capacity. Therefore, there is a need for understanding the relative effects of specific cognitive characteristics (e.g., sustained attention capacity and reasoning ability) and psychomotor abilities (e.g., reaction time, coordination capacity, visual pursuit, and visual perception skills) on detecting the hazards on the road accurately.

Past research, especially on older drivers, has detailed the effects of cognitive and psychomotor capacities on driving and safety skills (e.g., Anstey, 2005). For instance, decision making and cognitive skills are accepted as the essential skills for both driving and hazard perception (Fitzgerald \& Harrison, 1999). Although the effects of cognitive, psychomotor, and hazard perception abilities on crash risk and/or driving safety have been extensively investigated, how and to what extent the specific cognitive and psychomotor capacities uniquely predict hazard perception skills have been rarely examined. Employing a large driver sample, the present study primarily aims to investigate the power of a number of cognitive and psychomotor abilities in predicting HPDA, which is the initial stage in hazard perception process. Secondly, in order to compare the relative role of these variables in predicting HPDA and more concrete indicators of risky driving, this study also aims to predict the critical indicators of risky driving (i.e., selfreported aberrant driver behaviors, the number of accidents involved, and the number of violation tickets) using the same set of cognitive and psychomotor variables. 
In sum, in the present study, the power of specific cognitive and psychomotor skills in predicting HPDA and the indicators of risky driving were examined by employing a series of sequential regression analyses after controlling for the effects of age, level of education, and annual km driven in the first step.

\section{METHOD}

\section{Participants and Procedure}

The sample consisted of professional male drivers $(\mathrm{N}=2541)$ who had applied to psychotechnical driver assessment centers located in the major cities in Turkey. The majority of the participants were young and middle-aged drivers (mean age $=38.29$, SD $=9.92$, range $=18-$ 74). Of the participants, $34 \%$ were elementary school, $57 \%$ were middle and high school, and $9 \%$ were university graduates. Following the completion of practice trials, drivers were administered a computer-based cognitive and psychomotor test battery in the driver assessment centers. Following the completion of the computerized tests, participants filled out a driver behavior measure, and filled out a demographic questionnaire assessing the history of road crashes and driving violation tickets.

\section{Instruments}

Instruments consisted of a questionnaire and the computerized cognitive and psychomotor test battery explained below. Computerized tests were originally developed by Sümer et al., (2002) to assess cognitive and psychomotor skills of the convicted drivers in Turkey.

Driver Hazard Perception Accuracy Test (DHPAT). The DHPAT is a computer-based test of hazard perception detection accuracy developed for the purpose of generating a real life hazard detection test. A number of 30 second long videos including different driving hazards and risks were produced using the recordings from the driver's point of view with a camera stabilized on the windshield of a car. All recordings were taken from the real traffic flows. Eight videos from a pool of recordings were selected by an expert panel and used in this test. As in the situation awareness assessment, drivers were asked to press a button on the steering wheel of the simulator as soon as they noticed an hazard, mistake of other drivers, or any risk on the road shown in the video and keep holding the hand on the button as long as the hazard (or risk, mistakes) last in the screen. One of the videos did not have any hazard and was used for control purposes. The duration of hazard in each video varied from 5 to 12 seconds. The duration in which actual presentation of hazard and "hand on button" overlap (i.e., the hazard perception detection duration) was used as an index of HPDA. Moreover, a second index, called hazard perception latency, was used. This index reflects lateness in responding to and/or failure to end responding to hazards. In other words, while the former index represents a HP detection capacity, the latter index indicates a failure or latency in accurately detecting hazards.

Monotonous Attention Test (MAT). The MAT is a computer-based cancellation task consisting of differently designed version of road traffic signs arranged in an "11X17" matrix. The task was to identify 50 items identical to the target item out of 186 items placed in the matrix. The 
participants are given a maximum of 2.5 minutes to complete the task. The number of correct (hits) responses in the MAT was used as a measure of monotonous attention in this study.

Selective Attention Test (SAT). The SAT consists of 60 items each having six traffic signs placed in " $4 \mathrm{X} 4$ " matrix of squares. Traffic signs are placed in the last three squares of the first row and column. The rest of the squares are unfilled. One of the traffic signs on the top (row) and very left column was identical to each other and the drivers are asked to find the identical signs and touch the crossing point (square) of these signs on the matrix. Each matrix is presented for 3 second. As in the MAT, the number of correct (hits) was used as the index of selective attention in this test

Visual Pursuit Test (VPT). The VPT is a type of visual attention test aiming to assess drivers' visual plasticity and tracking ability in a complex labyrinth task. In the VPT, there are 10 complex lines placed in a circle each starting from a different point on the edge of the circle. Drivers are asked to find the end points of the randomly presented lines by tracking with their eyes without using their hands. The number of the correct responses and the task completion time are calculated representing drivers' performance on the test. In this study, the duration of the task was used as the indicator of visual pursuit skill and thus high scores represent worse performance.

Visual Perception and Memory Skills Test (VP-MST). The VP-MST consists of 13 photographs taken from different road situations and was constructed as the picture stimulus of driver environment including vehicles, pedestrians, and/or traffic signs. Each picture was shown for 0.75 seconds and later five questions were asked regarding if they had seen any vehicles, pedestrians, and/or traffic signs in the picture. Response duration in answering the questions about each picture was calculated as the measure of visual perception and memory skills and thus higher scores represented lower levels of visual perception.

Hand-Eye Coordination Test (HECT). The HECT aims to assess drivers' motor coordination skill between the hands and eyes. Using a PC based simulator, drivers were asked to drive the car in both routine and complex road trails by trying to stay in the designated lane. Speed of the car was controlled by the computer. The duration drivers stay off the road in complex road environment was used as an indicator of hand-eye coordination ability. Therefore, higher scores represented lower levels of coordination skill.

Reaction Time (RT) Test. RT was measured by employing a series of compatible and controlled reactions to the given stimuli. Drivers were instructed to react by pressing a button as soon as they saw a green square or circle on the screen and not to respond when they saw the red ones. When they saw the circles, they were asked to press with their hands and when they saw squares, they were asked to press with their feet. Stimuli on the right and left side were responded with the corresponding hand or foot. Only reactions given with hands were calculated in this study and used as the reaction time latency score.

Measure of Aberrant Driving Behaviors and Demographic Questions: Participants also completed a 6-item self-reported aberrant driver behavior measure and demographic questionnaire including the questions about the number of traffic accidents they had been 
involved in the last five years and the number of traffic violation or offence tickets they had received in the last three years.

\section{RESULTS}

The scores on hazard perception detection accuracy and latency indices, and three variables reflecting risky driving (i.e., the average self reported aberrant behaviors, the number of road accidents in the last five years, and the number of traffic violation tickets in the last three years) were used as the criterion (dependent) variables and the indices of seven cognitive and psychomotor skills (i.e. monotonous attention, selective attention, reasoning, reaction time, handeye coordination, visual pursuit, and visual perceptual and memory skills) were used as the main predictors in the regression analyses. The three demographic variables: exposure to driving (km driven in a year), age, and level of education (year attending school) were used as the covariates.

A series of sequential regression analyses was conduced to test the power of seven cognitive and psychomotor skills in predicting these five outcome variables after controlling for the three demographic characteristics (age, education, and annual km driven). As seen in Table 1, after controlling for the demographic characteristics, the majority of the cognitive and psychomotor skills significantly predicted both HPDA and HP latency. Reasoning ability, selective attention, and visual pursuit with moderately strong beta values seem to be the critical components of HP. The three demographic variables explained only $1 \%$ of hazard detection accuracy capacity and $3 \%$ of hazard detection latency in the first step, and the cognitive and psychomotor skills explained the remaining $36 \%$ and $37 \%$ of the variance on these variables, respectively in the second step.

The same cognitive and psychomotor skills, however, weakly predicted self-reported aberrant driving behaviors, the number of accidents, and violation tickets taken. As would be expected, annual km driven (i.e., exposure to driving) was the only demographic variable in the first step that consistently and positively predicted these three outcome variables. Although the magnitude of the beta weights was small, visual perception and memory skills in the second step were the only significant predictors predicting all of the outcome variables. The set of predictors explained 5 to $13 \%$ of the variances in these three outcome variables representing driving risk.

In sum, findings suggested that although the size of their unique contribution varies, higher-order cognitive and psychomotor abilities were significantly associated with HPDA. Selective attention, reaction time, and visual pursuit capacities appeared to be moderately strong correlates of HPDA. These variables, however, were weakly associated with risky driving.

\section{DISCUSSION}

Cognitive and psychomotor processes including decision making capacity, attention, reaction time, and visual pursuit are integral aspects of hazard perception skills. Although there is now accumulated evidence on the effects of various cognitive and psychomotor skills on driving performance and safety, especially among older drivers, their effects on HPDA among young and professional drivers were understudied. The results of the current study showed that selective attention, reasoning, and visual pursuit are the relatively independent and moderately strong 
predictors of hazard perception detection accuracy on the road among professional drivers. Although the sample in this study was large and represents professional drivers in Turkey, it includes only six drivers over 65 years of age representing older drivers. Therefore, it was not possible to test if these skills become even more critical after certain age in predicting HPDA. Horswill et al. (2008) found that hazard perception ability declines with increasing age, even in a sample of older adults who see themselves healthy.

Table 1. Sequential regression analyses in predicting HPDA and risky driving variables $(\mathbf{N}=2541)$

\begin{tabular}{|c|c|c|c|c|c|}
\hline & $\operatorname{HPDA}(\beta)$ & $\operatorname{HPLI}(\beta)$ & $\operatorname{SRRD}(\beta)$ & RTA $(\beta)$ & $\operatorname{TICK}(\beta)$ \\
\hline \multicolumn{6}{|l|}{ Step 1} \\
\hline Age & $-.07 * *$ & $-.05 *$ & .03 & $.12^{* * *}$ & -.02 \\
\hline Education $^{1}$ & $.05 *$ & $.08 * * *$ & $.14^{* * *}$ & .04 & $.08^{* *}$ \\
\hline Exposure-annual km & -.01 & $.14^{* * *}$ & $.08^{* * *}$ & $.05 *$ & $.15^{* * *}$ \\
\hline $\mathrm{R}^{2}$ Change & .01 & .03 & .02 & .02 & .03 \\
\hline \multicolumn{6}{|l|}{ Step 2} \\
\hline Age & -.03 & $-.06 * *$ & .03 & .02 & -.02 \\
\hline Education & -.01 & $.11^{* * *}$ & $.11^{* * *}$ & .02 & $.06^{*}$ \\
\hline Exposure-annual km & .03 & $.09 * * *$ & $.09 * * *$ & $.06^{*}$ & $.16^{* * *}$ \\
\hline Monotonous Attention & $.10^{* * *}$ & .01 & .02 & $.05 *$ & .01 \\
\hline Selective Attention & $.17 * * *$ & .04 & $-.08 * * *$ & -.03 & $-.07 * *$ \\
\hline Reaction Time & $-.12 * * *$ & $.19 * * *$ & -.02 & -.01 & .01 \\
\hline Hand-Eye Coordination & $-.05^{*}$ & -.02 & -.01 & -.04 & -.03 \\
\hline Reasoning Ability & $.23 * * *$ & $-.21 * * *$ & $.07 * *$ & -.02 & .03 \\
\hline Visual Pursuit Skill & $-.17 * * *$ & $.27 * * *$ & $.14^{* * *}$ & .04 & $.09 * * *$ \\
\hline Visual Perception Skill & $-.07 * *$ & $.23^{* * *}$ & $.22 * * *$ & $.16^{* * *}$ & $.15^{* * *}$ \\
\hline $\mathrm{R}^{2}$ Change & .36 & .37 & $.11^{* * *}$ & .03 & .06 \\
\hline $\mathrm{R}^{2}$ Total & .37 & .40 & .13 & .05 & .09 \\
\hline
\end{tabular}

Considering that cognitive and psychomotor abilities moderately strongly predicted the indictors of hazard perception skills and weakly predicted the indicators of risky driving, future studies should also test if hazard perception skills mediate the link between these higher order cognitive/psychomotor abilities and driving risk.

Future research should also examine how driving experience (novice vs. experienced drivers) and age (young vs. elderly drivers) moderate the link between these cognitive and psychomotor abilities and hazard perception detection and implementation skills.

These findings underscore the role of higher order perceptual and cognitive processes, especially selective attention and reasoning, underlying hazard perception ability and have implications for driver assessment, training, and cognitive demands for driving. 


\section{ACKNOWLEDGMENT}

The author would like to thank ALG Driver Psycho-Technical Driver Assessment Company, Sevil Ipekkan, and Pinar Cinar who provided the data used for this analysis.

\section{REFERENCES}

Anstey, K. J., Wood, J., Lord, S., \& Walker, J. G. (2005). Cognitive, sensory and physical factors enabling driving safety in older adults. Clinical Psychology Review, 25, 45-65.

Banbury, S., \& Tremblay, S. (2004). A cognitive approach to situation awareness: Theory, measurement and application. Aldershot, UK: Ashgate Publishing.

Deery, H. A. (1999). Hazard and risk perception among young novice drivers. Journal of Safety Research, 30, 225-236.

Fitzgerald, E.S., \& Harrison, W.A. (1999). Hazard perception and learner drivers: A theoretical discussion and an in-depth survey of driving instructors. Monash University Accident Research Centre, Report No. 161.

Groeger, J. A. (2000). Understanding driving: Applying cognitive psychology to a complex everyday task. Philadelphia: Psychology Press.

Helman, S. (2008). Situational judgment in driver training and assessment: a literature review (PPR312). Published Project Report. Wokingham, Berkshire: Transport Research Laboratory (TRL).

Horswill, M.S., Marrington, S.A., McCullough, C.M., Wood, J., Pachana, N.A., McWilliams, J., et al. (2008). The hazard perception ability of older drivers. Journals of Gerontology: Series B: Psychological Sciences and Social Sciences 63B (4), 212-218.

Horswill, M. S., \& McKenna, F. P. (2004). Drivers’ hazard perception ability: Situation awareness on the road. In S. Banbury \& S. Tremblay (Eds.), A cognitive approach to situation awareness: Theory and application (pp. 155-175). Aldershot, England: Ashgate.

Horswill, M.S., Pachana, N.A., Wood, J., Marrington, S.A., McWilliams, J., McCullough, C.M., (2009). A comparison of the hazard perception ability of matched groups of healthy drivers aged 35 to 55, 65 to 74, and 75 to 84 years. Journal of the International Neuropsychological Society 15, 799-802.

McKenna, F. P., \& Horswill, M. S. (1999). Hazard perception and its relevance for driver licensing. Journal of the International Association of Traffic and Safety Sciences, 23(1), 2641.

Sümer, N., Ayvasik, B., \& Er, N. (2002). Driver Psycho Technical Evaluation Handbook and Norm Values. [Surucu Psikoteknik Degerlendirme Sistemi Kullanici El Kitabi ve Norm Degerleri]. Mobilsoft Mobile Information and Communication Technologies A.S. 